Research Paper

\title{
Suppression of hypoxia-induced excessive angiogenesis by metformin via elevating tumor blood perfusion
}

\author{
Ji-Chang Wang ${ }^{1,2, *}$, Guang-Yue $\mathrm{Li}^{3, *}$, Ping-Ping Lij ${ }^{2}$, Xin Sun ${ }^{4}$, Wei-Ming Li ${ }^{1}$, Yan $\mathrm{Li}^{1}$, \\ Shao-Ying Lu ${ }^{1}$ and Pei-Jun Liu ${ }^{2}$ \\ ${ }^{1}$ Department of Vascular Surgery, First Affiliated Hospital of Xi'an Jiaotong University, Xi'an, Shaanxi Province, 710061, \\ P.R.China \\ ${ }^{2}$ Center for Translational Medicine, First Affiliated Hospital of Xi'an Jiaotong University, Xi'an, Shaanxi Province, 710061 , \\ P.R.China \\ ${ }^{3}$ Department of Science and Technology, First Affiliated Hospital of Xi'an Jiaotong University, Xi'an, Shaanxi Province, \\ 710061, P.R.China \\ ${ }^{4}$ Department of Thoracic Surgery and Oncology, First Affiliated Hospital of Xi'an Jiaotong University, Xi'an, Shaanxi Province, \\ 710061, P.R.China \\ *These two authors contributed equally to this work and share co-first authors \\ Correspondence to: Pei-Jun Liu, email: liupeijun@mail.xjtu.edu.cn \\ Shao-Ying Lu, email: robertlu@mail.xjtu.edu.cn
}

Keywords: metformin, inhibition of tumor angiogenesis, hypoxia, elevating blood perfusion, HIF-1a

Received: April 09, $2017 \quad$ Accepted: May 09, $2017 \quad$ Published: May 19, 2017

Copyright: Wang et al. This is an open-access article distributed under the terms of the Creative Commons Attribution License 3.0 (CC BY 3.0), which permits unrestricted use, distribution, and reproduction in any medium, provided the original author and source are credited.

\section{ABSTRACT}

The anti-diabetic metformin has been demonstrated to be effective in suppression of tumor progression via multiple mechanisms, in which angiogenic inhibition is involved. Hypoxia is a common feather of malignant tumor and promotes angiogenesis via induction of pro-angiogenic factors. However, the effect of metformin on tumor hypoxia and the association with angiogenic inhibition are still unclear. In the current study, we investigated the effects of metformin on both tumor blood perfusion and hypoxia-induced excessive angiogenesis. In the tumor region adjacent to necrosis, aberrantly excessive angiogenesis resulted from hypoperfusion-induced intense hypoxia and greatly contributed to the high average levels of both microvessel density and vascular branch density. Metformin administration increased the percentage of lectin-perfused vessels and reduced hypoxyprobe-positive area. This metformin-induced amelioration of hypoxia was accompanied by a significant reduction in expressions of both HIF-1a and angiogenesis-associated factors (AAFs). Consequently, inhibited excessive angiogenesis in hypoxic peri-necrotic region was observed in metformin-treated tumor. Further stable knockdown of HIF-1a abrogated hypoxia-induced AAFs in vitro and reduced both microvessel density and area of fitc-conjugated dextran that leaked outside the vascular lumen. Taken together, metformin ameliorated tumor hypoxia and restrained HIF-1a-induced expressions of AAFs through elevating tumor blood perfusion, thus suppressing the excessive tumor angiogenesis.

\section{INTRODUCTION}

Malignant tumor is one of the disorders characterized by abnormal or excessive angiogenesis [1], which has been deeply implicated in cancer progression. When dysregulated, aberrantly formed tumor vessels by angiogenesis are always tortuous, dilated and excessively branched [2]. Consequently, this disorganized vasculature is inefficient for blood supply and lead to a hypoxic tumor microenvironment that has a fundamental role for cancer progression [3]. Suppression of tumor angiogenesis by molecular inhibitors has provided new opportunities for cancer management and greatly broadened our understanding of the role of angiogenesis 
in tumor progression. However, current anti-angiogenesis treatments, in which vascular endothelial growth factor (VEGF) inhibitors are key components, have been greatly challenged for therapeutic resistance [4] and induction of metastasis [5]. Thus, from drug discovery standpoint, a promising solution in overcoming this challenge is to explore new drug or new aspects of old drugs.

Epidemiological and experimental studies have revealed that the anti-diabetic metformin was able to reduce risk of various types of cancer [6] and inhibit tumor progression [7]. In the past few decades, metformin was extensively applied for the management of diabetes and its good tolerance has been deeply verified in the longterm clinical use. Due to its clinical safety and anti-tumor activities demonstrated by pre-clinical researches $[8,9]$, researchers are increasingly concerned to understand its anti-tumor potentials. Recently published articles have expanded this mechanistic profile by providing the novel insight into angiogenic suppression $[10,11]$. However, the mechanism underlying metformin-induced angiogenesis inhibition remains an enigma.

In this paper, efforts were devoted to investigate the effects of metformin on hypoxia-induced angiogenesis for various reasons. First, metformin has been shown to inhibit hypoxia inducible factor 1 alpha (HIF-1 $\alpha$ )-VEGF signaling, but the association with HIF- $1 \alpha$-induced other AAFs than VEGF is still undefined. Second, hypoxia induces angiogenesis by stimulating the secretion of angiogenesisassociated factors (AAFs) [12]. However, it is unclear whether metformin inhibits angiogenesis by ameliorating tumor hypoxia. Third, tumor starts to experience hypoxia when size reach $2 \mathrm{~mm}^{2}$ and hypoxia induces HIF-1 $\alpha$ accumulation. Although metformin had been shown to increase tumor oxygenation [13], it remains incompletely understood about whether metformin inhibits HIF-1 $\alpha$ induced angiogenesis by increasing tumor blood perfusion.

In the present study, we investigated the effects of metformin on inducing anti-angiogenesis in different tumor regions, especially in peri-necrotic region (PNR) that has been demonstrated to be hypoxic. We used 4T1 murine breast cancer cell, which would encounter hypoxia and necrosis in vivo [14], to investigate if metformin could be used for inhibition of hypoxia-associated angiogenesis. Our results of both in vivo and in vitro experiments showed that metformin restrained hypoxia-induced excessive angiogenesis in PNR by suppressing HIF-1 $\alpha$-induced proangiogenic factors via elevating tumor blood perfusion.

\section{RESULTS}

\section{Excessive angiogenesis occurred in tumor region adjacent to necrosis}

Derived from a mouse mammary gland cancer [16], 4T1 cell line could be orthotopically transplanted into the BALB/c mouse [14], which has a normal immune system. According to different angiogenic abilities of vessels (Figure 1A-1C), 4T1 tumor was divided into perinecrotic region (PNR), transitional region (TR) and nonnecrotic region (NNR). IHC staining for CD31, a specific endothelial marker, showed that tumor region adjacent to necrosis exhibited an aberrantly high microvessel density (MVD) and vascular branch density. Decreased CD31 expression of tumor vessels was found in the edge region of necrosis (Red arrows; Figure 1B). Critically, vessels in PNR and TR had higher vascular abilities of both sprouting and branching than those in NNR (Figure 1A-1E). These results indicate that the closer the vessel lies to necrosis, the more excessive angiogenic phenotype is likely to be observed.

\section{MVD in PNR was linearly correlated with necrotic area}

PNR had a higher MVD than average MVD of the whole tumor, while NNR had a lower MVD (Figure 1E), suggesting excessive angiogenesis in PNR contributed to high MVD of the whole tumor. Since hypoxia induces tissue necrosis [17], we next investigated whether tumor necrosis was correlated with excessive angiogenesis in peri-necrotic region. Results of spearman linear correlation analysis showed (Figure 1F-1I) that both average MVD and PNR MVD had significant linear correlation with percentage of necrotic region in untreated 4T1 cancer. Critically, PNR MVD exhibited a higher statistical significance than average MVD. However, MVD of both NNR and TR had no linear correlation with percentage of necrotic region. Spearman $\mathrm{R}$ for PNR MVD was $0.825(P=0.0033)$; spearman $\mathrm{R}$ for average MVD was $(P=0.029)$; spearman $\mathrm{R}$ for TR MVD was $0.432(P=0.2124)$; spearman $\mathrm{R}$ for NNR MVD was $0.2622(P=0.4643)$. These data indicated that correlation between average MVD and tumor necrosis resulted from PNR MVD but not MVD of TR and NNR. This speculation was further supported by the fact that only the correlation between PNR MVD and average MVD was statistically significant (Supplementary Figure 1A-1C).

\section{Hypoperfusion resulted in hypoxia-induced excessive angiogenesis in PNR}

To investigate the relationship between perfusion status and hypoxia-associated angiogenesis, 4T1 tumor bearing-mice were intravenously injected with TRITCconjugated lectin and PIMO (for hypoxia detection). Intravenously injected lectin is able to bind to endothelial cell (EC), thus labeling the blood-perfused vascular endothelial layer. Further immunofluorescent staining (Figure 2A, 2B) showed that vessels in both tumor edge and non-necrotic regions could be perfused by lectincontained blood, indicating a good blood perfusion status in both regions. However, only $1.72 \%$ vessels 
were labelled by intravenously injected lectin in the perinecrotic region (Figure 2C, 2D), where severe hypoxia was also found (indicated by positive hypoxyprobe signal). Furthermore, percentage of hypoxic tumor area had significantly linear correlation with both MVD and vascular branch density in PNR (Figure 2E, 2F). Spearman R for PNR MVD was $0.637(P=0.0476)$; spearman R for PNR vascular branch density was $0.797(P=0.0058)$. These data demonstrate hypoperfusion-induced hypoxia leads to excessive angiogenesis.
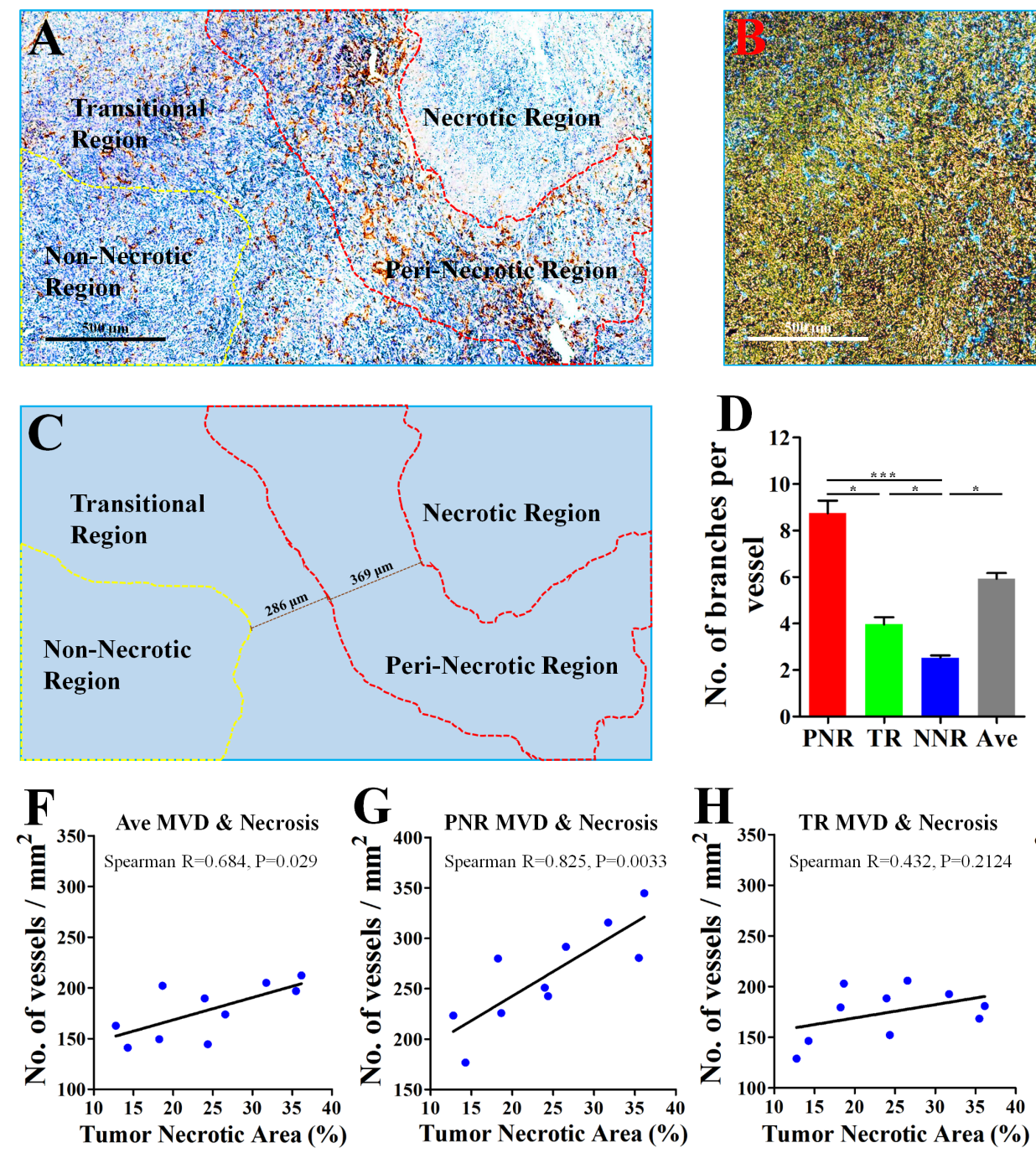

\section{Metformin reduced necrosis, inhibited angiogenesis and promoted vascular pericyte coverage}

Vascular pericyte coverage is an important marker for vascular maturity [18, 19]. As vascular maturity is the basis for vascular function [20], we next focused on pericyte coverage by vascular smooth muscle cells (VSMCs).We performed 3D-reconstruction of immunofluorescent double-staining for CD31 and $\alpha$-SMA
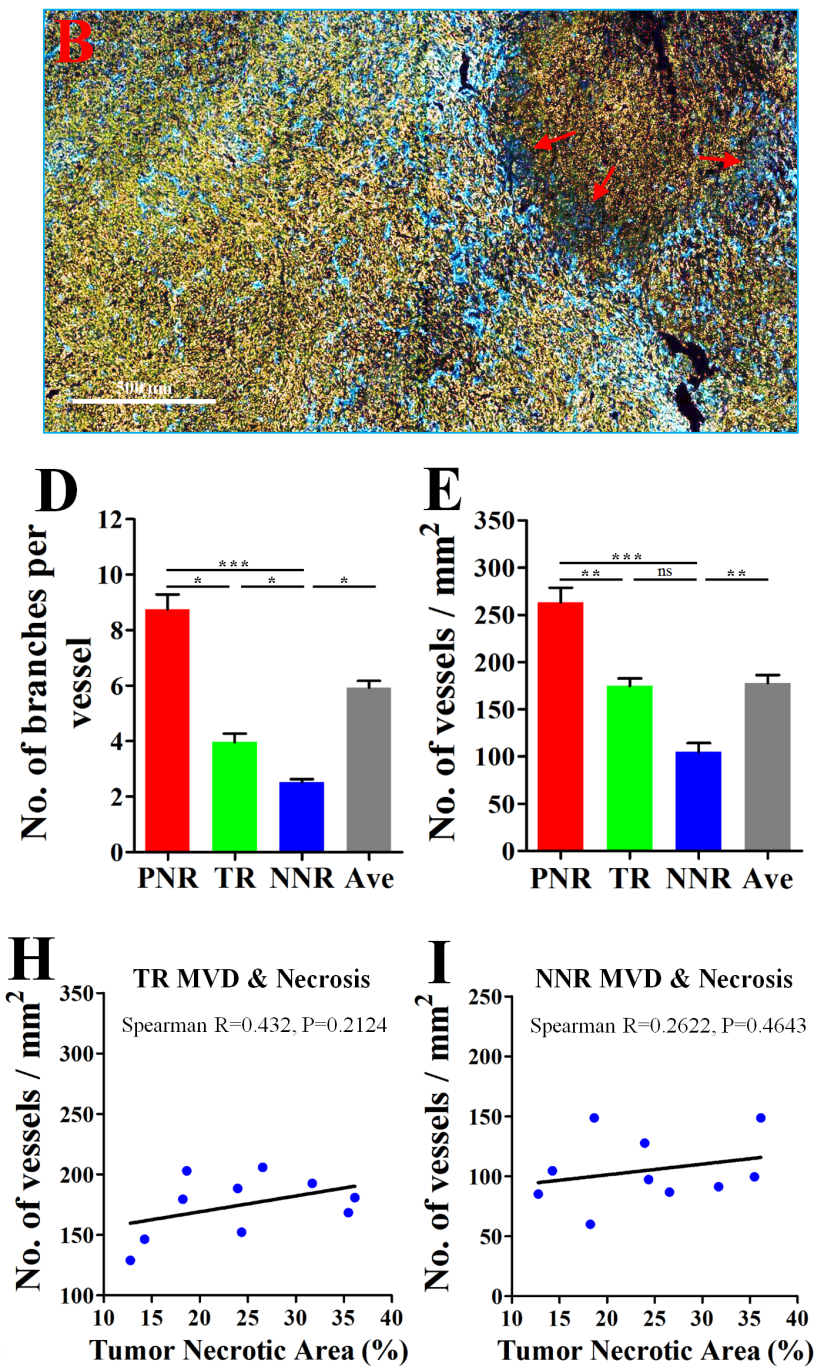

Figure 1: Excessive angiogenesis occurred in the peri-necrotic tumor region. 4T1 breast cancer cells were orthotopically inoculated into the fat pad of 4th breast of BALB/c mice. 28 days later, the whole tumor tissue from untreated mouse was extracted for further analysis. (A) Representative image (Immunological Histological Chemistry) for showing CD $31^{+}$vessels distributed in peri-necrotic region, transitional region and the non-necrotic region. Scale bar: $500 \mu \mathrm{m}$. (B) The color-reverse image and (C) regional distribution map of panel (A) for revealing the discrepancy of angiogenic ability of vessels distributed in different tumor regions. In the area adjacent to tumor necrosis, vessels exhibited the abnormally high abilities of sprouting and branching. Red tri-angle indicates vessels with decreased CD31 expression in the edge region of tumor necrosis. Scale bar: $500 \mu \mathrm{m}$. The peri-necrotic and non-necrotic regions were surrounded by red and yellow dotted lines, respectively. Quantification of (D) microvessel density (MVD, No. of vessels per mm $^{2}$ ) and (E) vascular branch density (No. of vascular branches per vessel) of regions having different distance from tumor necrosis $(n=10)$. Spearman analysis for evaluation of the linear correlation between percentage of tumor necrotic area and MVD of (F) the whole tumor area, (G) peri-necrotic region (PNR), (H) transitional region (TR) and (I) non-necrotic region (NNR). Quantitative data are indicated as mean \pm SEM. ${ }^{*} p<0.05$; $* * p<0.01$; $* * * p<0.001 ; \mathrm{ns}$ indicates no significant $(P>0.05)$. 

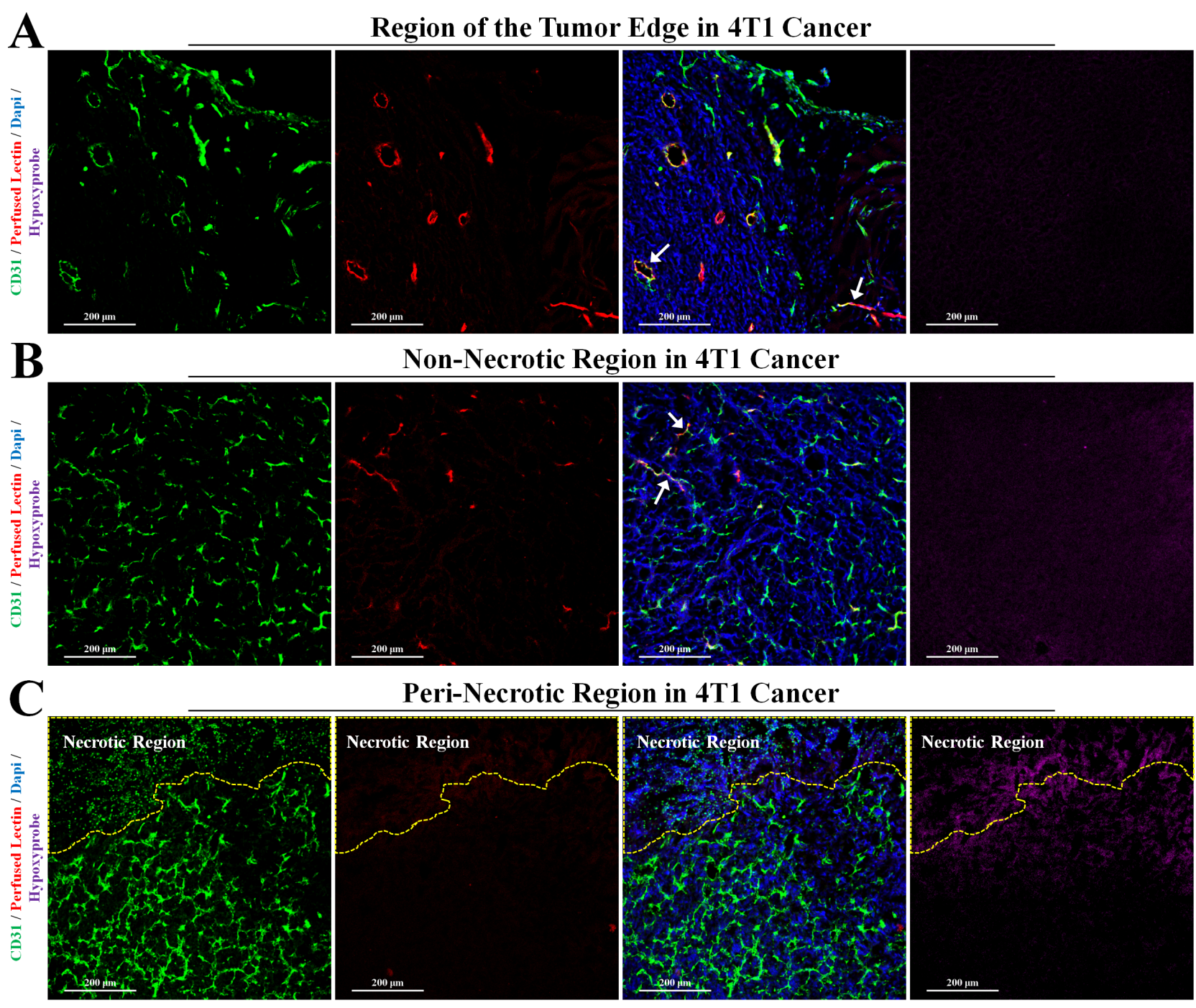

Peri-Necrotic Region in 4T1 Cancer
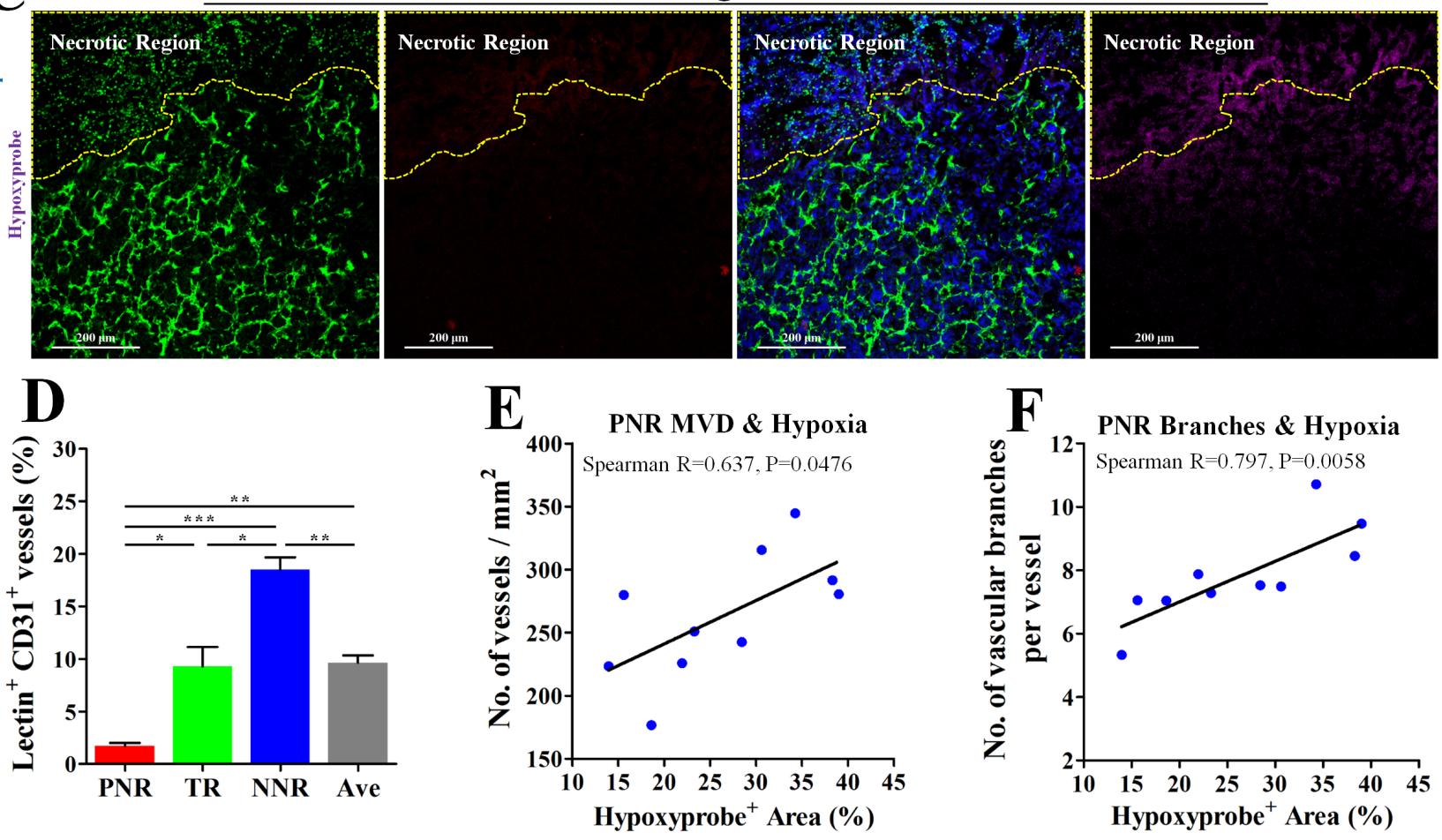

Figure 2: Hypoperfusion resulted in tumor necrosis and hypoxia-associated excessive angiogenesis in peri-necrotic region. The wholly extracted $4 \mathrm{~T} 1$ tumors were fixed by $4 \%$ paraformaldehyde and cut into $6 \mu \mathrm{m}$ thick frozen section for further immunofluorescent staining. $2 \mathrm{~h}$ and $15 \mathrm{~min}$ before extraction, pimonidazole hydrochloride and TRITC-conjugated lectin was injected into mouse tail vein, respectively. Staining for CD31 (Green), TRITC-conjugated lectin (Red) and pimonidazole hydrochloride (Violet) in (A) tumor edge, (B) non-necrotic and (C) peri-necrotic regions of 4T1 tumors from untreated BALB/c mouse. The necrotic region was surrounded by yellow dotted line. Scale bar: $200 \mu \mathrm{m}$. (D) Percentage of perfused-lectin ${ }^{+} \mathrm{CD} 31^{+}$vessels in all CD31 ${ }^{+}$vessels of the whole tumor area (Ave indicates average perfusion level), peri-necrotic region (PNR), transitional region (TR) and non-necrotic region (NNR). $n=10$. (E and $\mathbf{F}$ ) Spearman analysis for evaluation of the linear correlation between percentage of hypoxic area (detected by IHC staining for hypoxyprobe) and (E) MVD or (F) vascular branch density in peri-necrotic region (PNR). Quantitative data are indicated as mean \pm SEM. ${ }^{*} p<0.05 ; * * p<0.01 ; * * *<0.001$; ns indicates no significant $(P>0.05)$. 
(alpha smooth muscle actin), a specific marker for VSMC. In PNR, irregular vascular lumens were almost uncoated by VSMCs (Figure 3A), indicating a complete absence of pericyte coverage on vessels in PNR. Unlike tumor vessels, vessels distributed in normal breast tissue of BALB/c mouse were well coated by VSMCs (Figure 3B), suggesting a mature vasculature.

To study the effect of metformin on tumor vasculature, mice were orally administrated with metformin. Compared to untreated tumor (Figure 3A), metformintreated tumor exhibited a significantly promoted vascular maturity (Figure 3C, 3D). The change was indicated by increased ratio of co-located $\alpha$-SMA signal to CD31 signal in PNR, TR and NNR (Figure 3D). Furthermore, metformin apparently reduced tumor necrosis, MVD and vascular branch density in PNR (Figure 3E-3J), while having no effect on angiogenesis in both TR and NNR (Figure 3E-3J). Thus, metformin-induced reduction in average MVD and vascular branch density of whole tumor region mainly resulted from significantly inhibited angiogenesis in PNR.

\section{Metformin suppressed HIF-1a-induced AAFs in PNR}

HIF- $1 \alpha$ is one of the most critical factors for mediating angiogenesis in hypoxic condition [21, 22]. To explore the mechanism leading to inhibition of angiogenesis in PNR, we next investigated the change of HIF- $1 \alpha$ and AAFs. Further characterization of IHC staining showed that HIF-1 $\alpha$, VEGF, fibroblast growth factor-2 (FGF-2) and platelet-derived growth factor-B (PDGF-B) were highly expressed in the edge region of necrosis (Figure 4A), while angiopoietin-1 (Ang-1), angiopoietin-2 (Ang-2) and placental growth factor (PlGF) exhibiting weaker expression level. Further, to determine whether metformin-induced inhibition of PNR angiogenesis resulted from down-regulated AAFs, we focused on the change of AAFs in PNR. As expected, expression levels of HIF-1 1 and AAFs, including VEGF, PDGF-B, FGF-2 and PIGF, were significantly reduced in PNR of metformin-treated 4T1 tumor tissue. (Figure 4B) However, both Ang-1 and Ang-2 were not affected by metformin administration.

\section{Tumor hypoxia was ameliorated by metformin via elevating tumor blood perfusion}

In light of the reports that metformin has the potential to improved tumor oxygenation [13], we considered the possibility that metformin-induced inhibition of hypoxic angiogenesis might operate via increase of blood perfusion. Interesting, the unperfused condition of vessels in PNR was apparently reversed by a continuous administration of metformin (Figure 1C and Figure 5A), which was consistently accompanied by a significant reduction in hypoxia (Figure 5B). Further characterization of tumor blood perfusion showed that metformin increased average perfusion via elevation of perfusion in both TR and PNR (Figure 5C).

\section{Normoxia abrogated hypoxia-induced expressions of pro-angiogenic factors of 4T1 cancer cell in vitro}

To simulate the in vivo tumor hypoxia, 4T1 cancer cells were cultured under $21 \% \mathrm{O}^{2}$ and $1 \% \mathrm{O}^{2}$, respectively, and then mRNA levels of AAFs were detected by real-time quantitative PCR. As shown in Figure 5D, high mRNA expression levels of pro-angiogenic factors, including VEGF, FGF-2, PDGF-B, PlGF and Ang-2, in 4T1 cells cultured in hypoxic condition were significantly reduced by normoxia. Unsimilarly to pro-angiogenic factors, Ang-1, an anti-angiogenic factor, exhibited an increased mRNA level in normoxic condition. Thus, it appears that increased blood perfusion is able to ameliorate hypoxiainduced expressions of pro-angiogenic factors.

\section{Knock-down of HIF-1 $\alpha$ abrogated hypoxia- induced angiogenesis and reduced vascular leakage}

To further delineate the role of HIF-1 $\alpha$ in mediating hypoxia-induced angiogenesis, we established the 4T1 cell line with stable HIF-1 $\alpha$ knock-down. The knock-down efficiency was first verified in vivo. Our results of IHC staining for HIF-1 $\alpha$ (Figure 6A-6C) showed that HIF$1 \alpha$ expression was significantly inhibited in PNR, while HIF-1 $\alpha$ of NNR exhibiting slight reduction. These data indicated a successful knock-down of HIF-1 $\alpha$ in vivo. Furthermore, knock-down of HIF-1 $\alpha$ also significantly abrogated hypoxia-induced expressions of AAFs of $4 \mathrm{~T} 1$ cells both in vitro and in vivo (Figure 6D, 6E and Supplementary Figure 1D, 1E).

Given aberrant tumor vessel is characterized by intense vascular leakage, we next investigated whether blockade of HIF-1 $\alpha$ would reduce the vascular leakage, which was indicated by fitc-conjugated dextran leaking outside the vascular lumen. We found that dextran ${ }^{+}$area distributed outside tumor vessel was significantly reduced by $7.32 \times 10^{4}$ pixels in shRNA-HIF-1 $\alpha-4 \mathrm{~T} 1$ tumor, suggesting an inhibitory effect of HIF-1 $\alpha$ blockade on vessel leakage. (Figure 6F-6G) Moreover, vessels of shRNA-HIF-1 $\alpha-4$ T 1 tumor exhibited apparently weaker ability of angiogenesis in PNR than shRNA-control-4T1 tumor. (Figure 6F and $6 \mathrm{H}$ ) Taken together, these results show that hypoxia-induced HIF-1 $\alpha$ promotes aberrant angiogenesis in PNR by up-regulation of pro-angiogenic factors. Amelioration of tumor hypoxia by metformin treatment suppresses excessive angiogenesis via inhibition of HIF-1 $\alpha$-AAFs signaling. The associated mechanism responsible for metformin-induced inhibition of excessive angiogenesis is illustrated in Figure 7. 
A

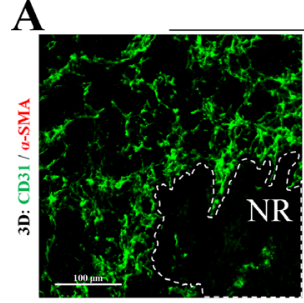

Untreated 4T1 Cancer
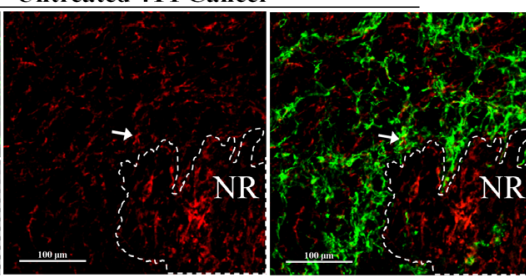

C
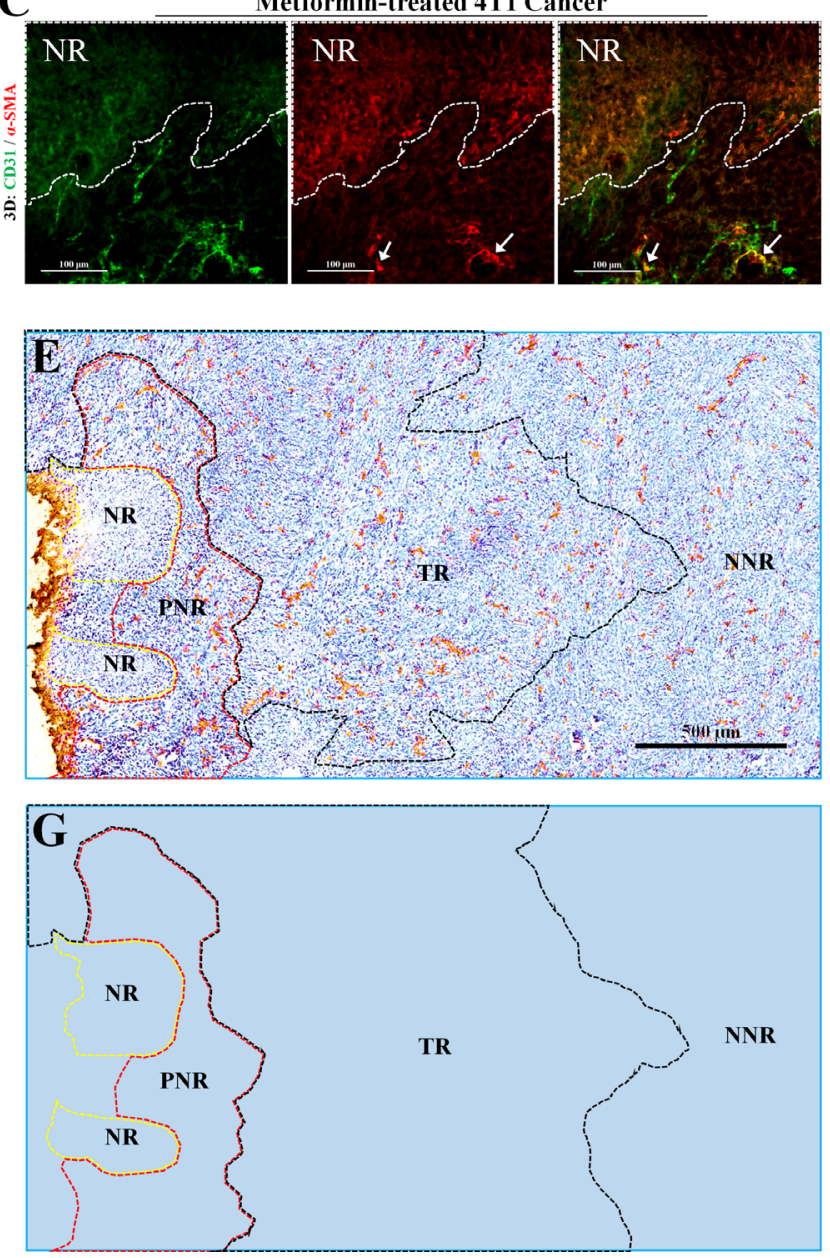

B
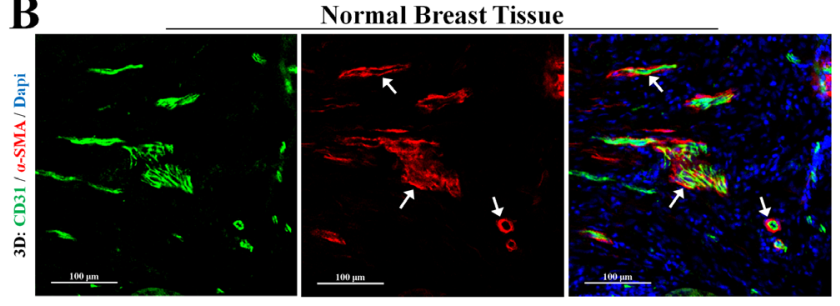

D

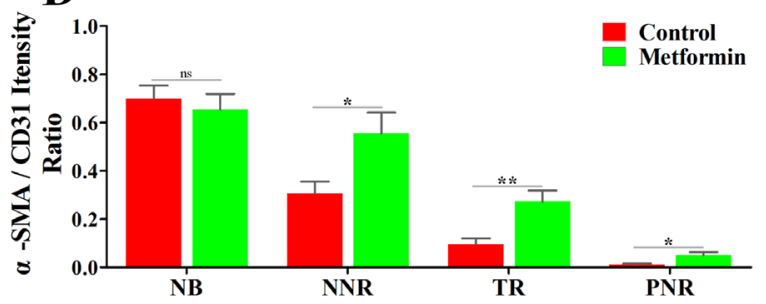

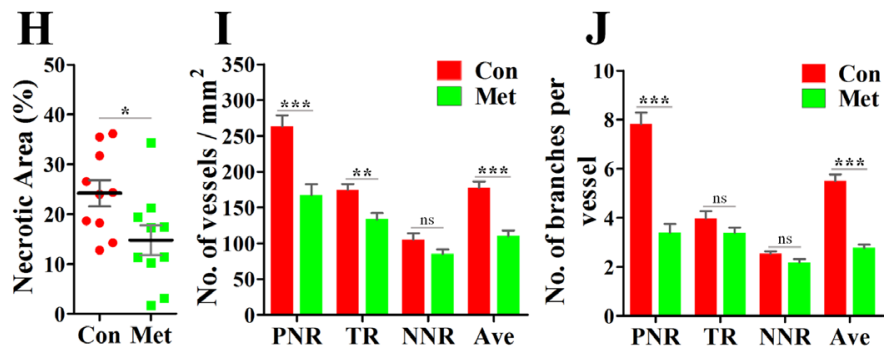

Figure 3: Increase of vascular maturity contributed to metformin-induced reduction of necrosis and MVD. 4T1 tumorbearing BALB/c mice were orally treated with metformin $(1.5 \mathrm{mg} / \mathrm{mL})$ for 21 days before extraction. The wholly extracted 4T1 tumors were fixed by $4 \%$ paraformaldehyde and cut into $40 \mu \mathrm{m}$ thick frozen section for further 3D-reconstruction of immunofluorescent signal. 3D-reconstruction of fluorescent signal of CD31 (Green) and $\alpha$-SMA (indicating vascular smooth muscle cell, VSMCs) in (A) untreated 4T1 tumor (Con), (B) normal breatst tissue and (C) metformin-treated 4T1 tumor (Met). $\alpha$-SMA signal co-located with CD31 signal indicate VSMCs that covered the tumor vascular lumen. (D) Quantification of ratio of co-located fluorescent $\alpha$-SMA to CD31 in NB (normal breast), PNR (peri-necrotic region), TR (transitional region) and NNR (non-necrotic region). Scale bar: $100 \mu \mathrm{m}$. (E) Representative image (Immunological Histological Chemistry) for showing CD31 ${ }^{+}$vessels distributed in peri-necrotic region, transitional region and non-necrotic region of metformin-treated 4T1 tumor. Scale bar: $500 \mu \mathrm{m}$. (F) The color-reverse image and (G) regional distribution map of panel (E) for revealing angiogenic ability of vessels distributed in different regions of metformin-treated tumor. Scale bar: $500 \mu \mathrm{m}$. The peri-necrotic and non-necrotic regions were surrounded by red and yellow dotted lines, respectively. (H) Quantification of percentage of necrotic area in 4T1 tumors from untreated or metformin-treated BALB/c mice $(n=10)$. Quantification of $(\mathbf{I})$ microvessel density (MVD, No. of vessels per $\mathrm{mm}^{2}$ ) and (J) vascular branch density (No. of vascular branches per vessel) of different regions in 4T1 tumors from untreated or metformin-treated BALB/c mice $(n=10)$. Quantitative data are indicated as mean $\pm \mathrm{SEM}$. ${ }^{*} p<0.05 ;{ }^{* *} p<0.01 ; * * * p<0.001$; ns indicates no significant $(P>0.05)$. 

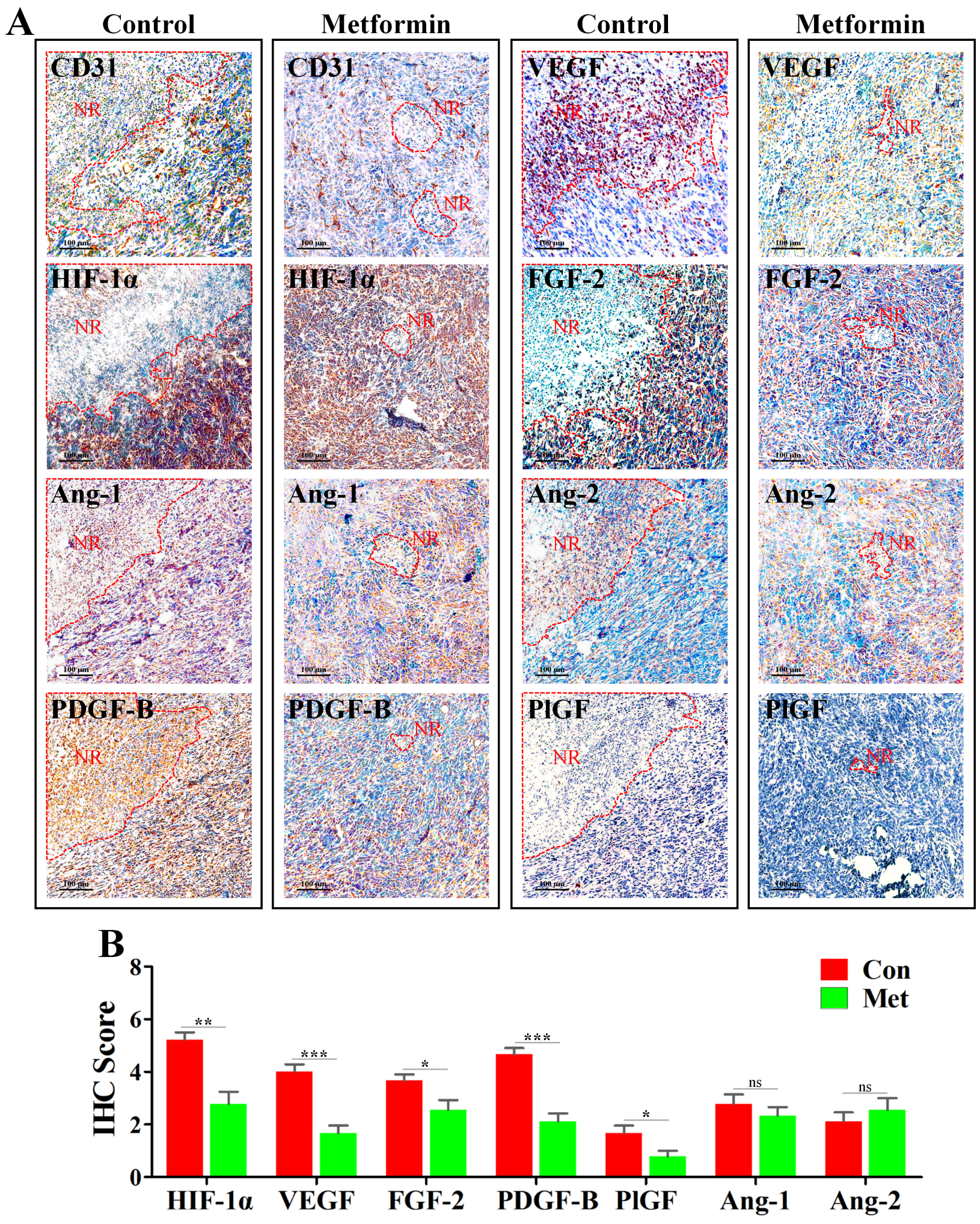

Figure 4: Metformin inhibited angiogenesis in peri-necrotic region by impeding HIF-1a-induced expressions of proangiogenic factors. 4T1 tumors extracted from untreated or metformin-treated mice were embedded in paraffin and cut into $4-5 \mu \mathrm{m}$ for IHC staining. (A) IHC staining for CD31, HIF-1 $\alpha$, Ang-1, Ang-2, PDGF-B, PIGF, VEGF and FGF-2 in untreated or metformin-treated BALB/c mice. Scale bar: $100 \mu \mathrm{m}$. Necrotic region is indicated by "NR" and its boundary is surrounded by red dotted line. (B) Quantification of IHC scores (addition of intensity score and positive signal area) of angiogenesis-associated factors, including HIF-1 $\alpha$, Ang-1, Ang-2, PDGF-B, PlGF, VEGF and FGF-2 $(n=10)$. Quantitative data are indicated as mean \pm SEM. ${ }^{*} p<0.05 ; * * p<0.01 ; * * *<0.001$; ns indicates no significant $(P>0.05)$. 


\section{DISCUSSION}

Herein, we report that metformin administration significantly inhibits excessive angiogenesis in murine breast cancer model. Support this conclusion comes from our observations that tumor angiogenesis resulted from hypoxia and was apparently inhibited by metformin via elevating tumor blood perfusion. Critically, metformininduced amelioration of hypoxia led to inhibited expressions of AAFs and consequently restrained angiogenesis in murine breast 4T1 carcinoma. Thus, unlike the direct drug activities reported in previously published articles [7, 23], in our study, hypoxic microenvironment was greatly contributed to the excessive and dysregulated angiogenesis, and we show that this was mediated by HIF- $1 \alpha$-induced expressions of AAFs, especially the proangiogenic factors.

Our results are consistent with the previous preclinical report of metformin-mediated amelioration of tumor hypoxia [13], and provided complementary information about why tumor oxygenation was elevated. By elevating tumor blood perfusion, more oxygenation is delivered to tumor region, especially the deep region, and lead to a corresponding reduction in tumor hypoxia. This result may reflect the greater dependence of abnormal tumor angiogenesis on hypoxia. Hypoxic tumor microenvironment correlates with advanced stage of the malignancies and greatly contributes to tumor resistance to anti-angiogenic, immune and chemical therapies $[2,24,25]$. Based on these evidences, reprogramming hypoxic tumor microenvironment has been widely considered as a promising therapeutic strategy [26, 27]. Thus, the therapeutic significance of metformin-induced amelioration of hypoxia should not be simply limited to inhibition of tumor angiogenesis.

Our results show that metformin is similar with the typical vascular-targeting agents in increasing blood perfusion [28], but dissimilar with the vascular-promoting therapy [29]. Although both therapies have similar effects on elevating blood perfusion, their influence on tumor vasculature is completely different. Vessels in tumors treated with vascular promoting agents were reported to be dilated and with leakage [30]. Our results showed that metformin-treated tumor exhibited a more mature vasculature, which was indicated by increased vascular pericyte coverage in peri-necrotic hypoxic region. Since
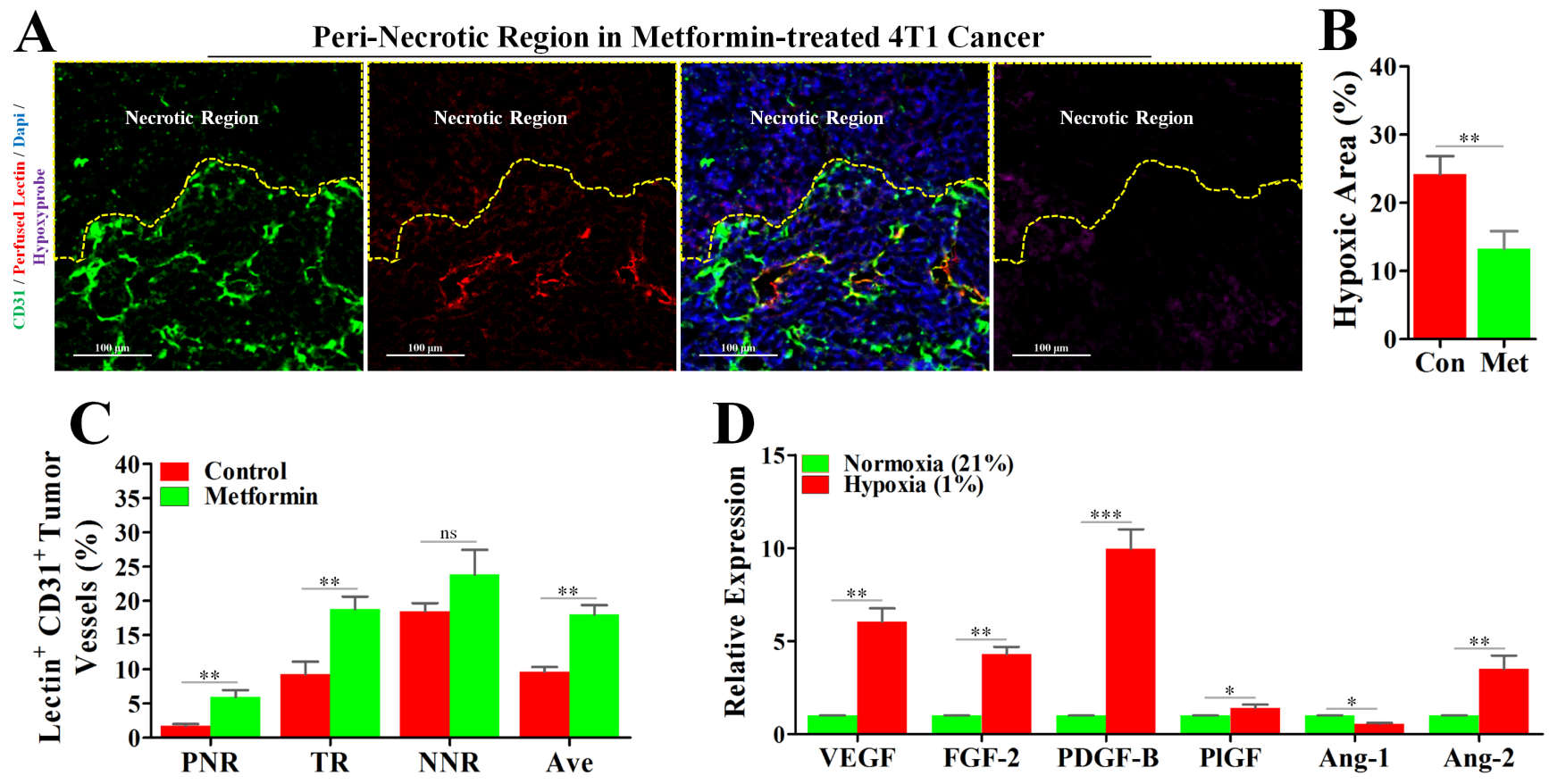

Figure 5: Metformin inhibited angiogenesis in peri-necrotic region by impeding HIF-1 $\alpha$-induced expressions of pro-angiogenic factors. 4T1 tumor-bearing mice were untreated (con) or persistently treated metformin $(1.5 \mathrm{mg} / \mathrm{mL}) \mathrm{for} 21 \mathrm{days}$. Before extraction, mice were sequentially injected with pimonidazole and TRITC-conjugated lectin. Staining for CD31 (Green), TRITCconjugated lectin (Red) and pimonidazole hydrochloride (Violet) in the peri-necrotic region of 4T1 tumors from metformin-treated BALB/c mouse. The necrotic region was surrounded by yellow dotted line. Scale bar: $100 \mu \mathrm{m}$. (B) Quantification of percentage of hypoxic area (indicated by hypoxyprobe positive area) in 4T1 tumors from untreated or metformin-treated BALB/c mice $(n=10)$. $(\mathbf{C})$ Percentage of perfused-lectin ${ }^{+} \mathrm{CD} 31^{+}$vessels in all CD31 ${ }^{+}$vessels of the whole tumor area (Ave indicates average perfusion level), peri-necrotic region (PNR), transitional region (TR) and non-necrotic region (NNR, $n=10)$. Mice were untreated or persistently treated with metformin. (D) Increased relative mRNA expressions (to normoxia) of various angiogenic factors of 4T1 cancer cells cultured in the hypoxic condition $\left(1 \% \mathrm{O}^{2}\right)$. mRNA level was detected by real-time quantitative PCR. Quantitative data are indicated as mean \pm SEM. ${ }^{*} p<0.05 ; * * p<0.01$; $* * * p<0.001$; ns indicates no significant $(P>0.05)$. 


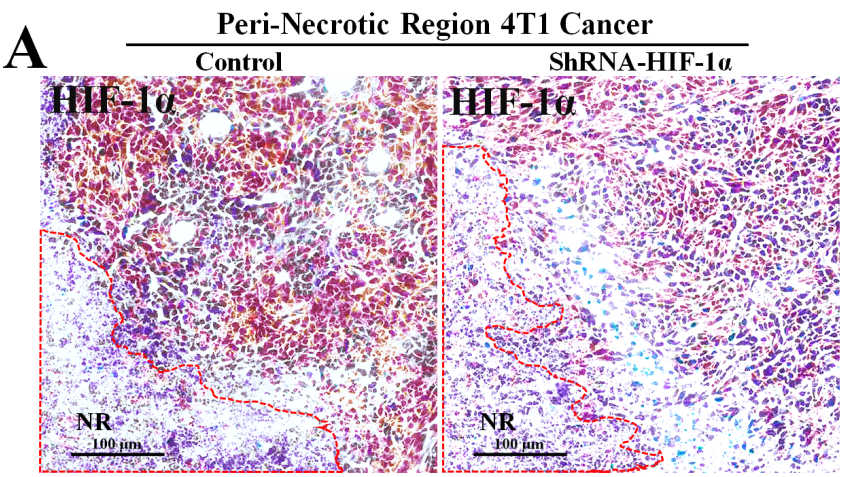

\section{C}

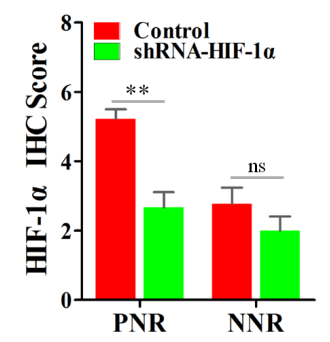

\section{D}

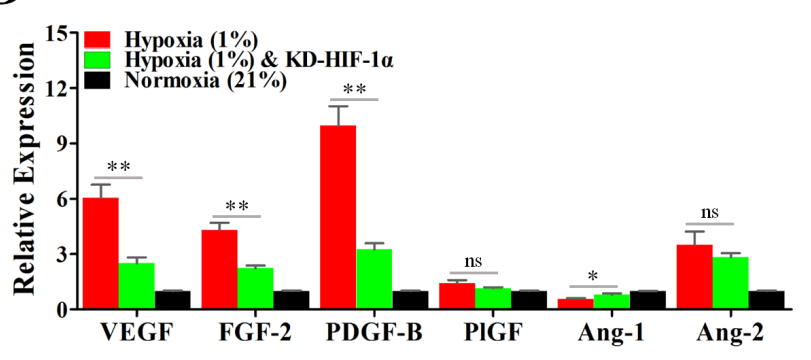

4T1-Control
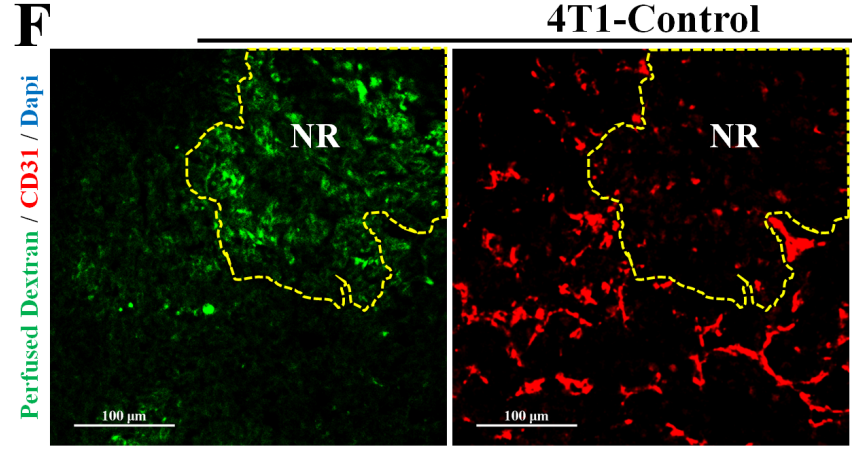

4T1-shRNA-HIF-1 $\alpha$
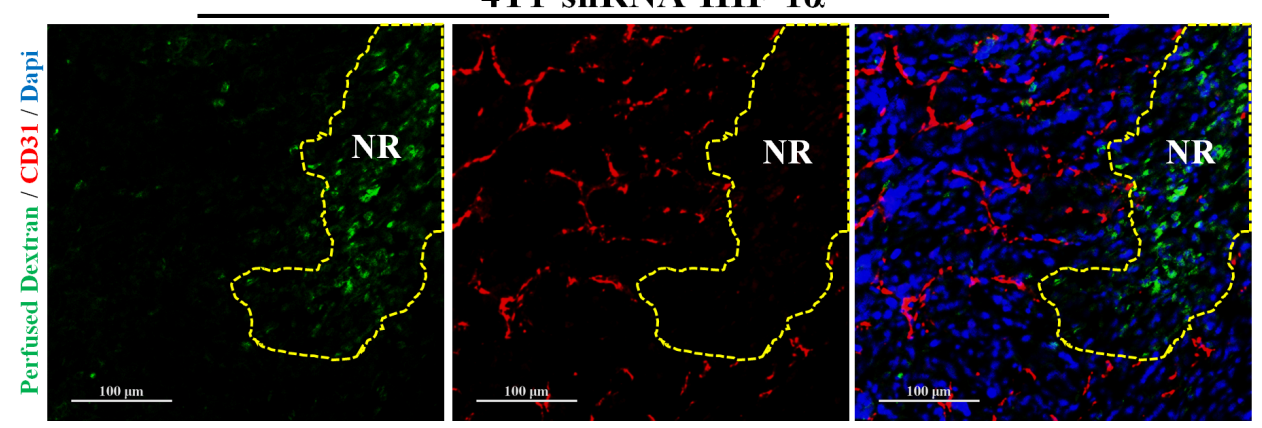
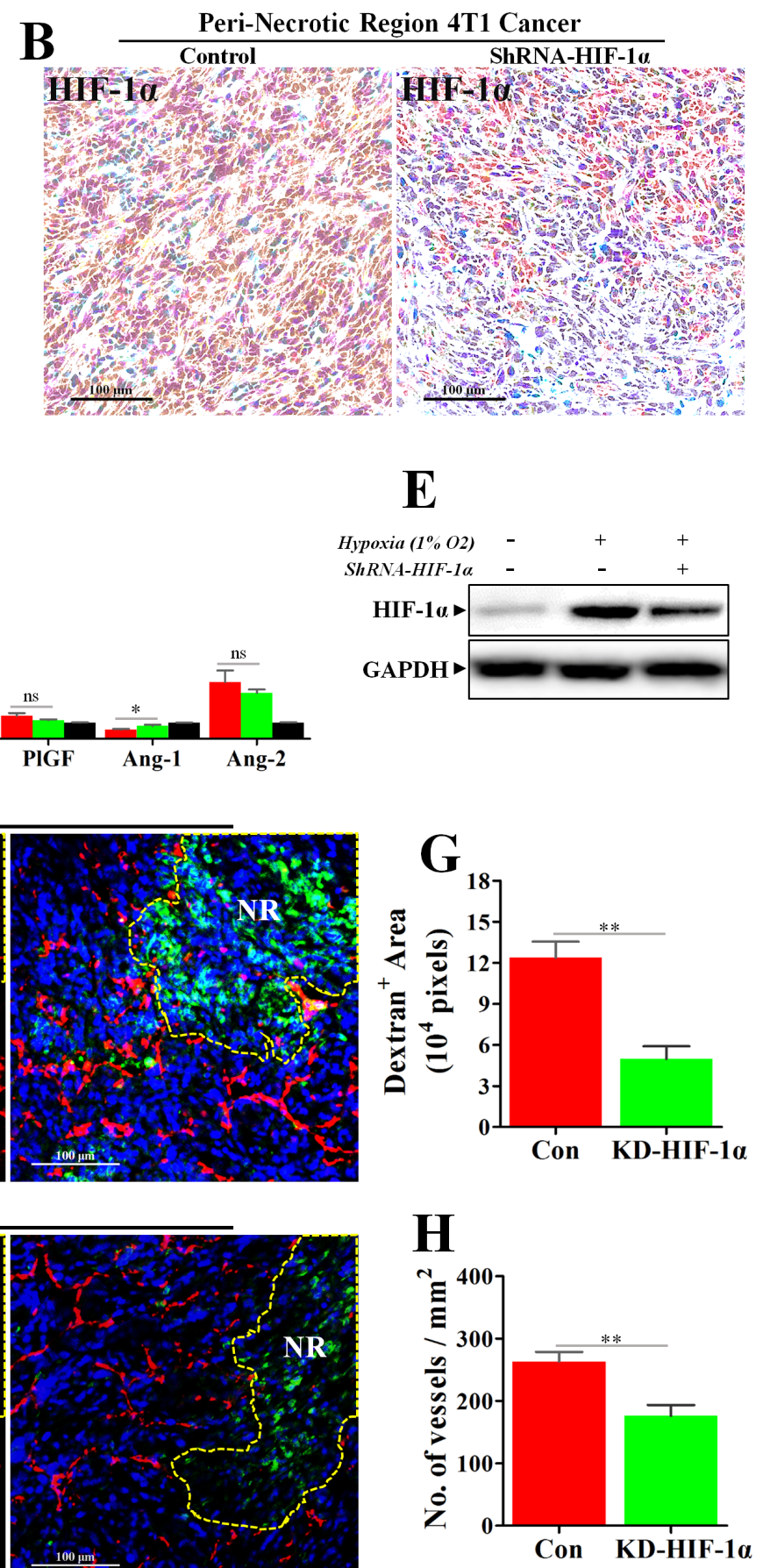

Figure 6: Knock-down of HIF-1 $\alpha$ inhibited hypoxia-induced abnormal angiogenesis and reduced vessel leakage. 4T1 cancer cells transfected with Lentivirus-shRNA-HIF-1 $\alpha$ or Lentivirus-shRNA-Con were orthotopically transplanted into the BALB/c mice. (A-C) IHC staining for HIF-1 $\alpha$, revealing a significant reduction of HIF-1 $\alpha$ protein level in both (A) peri-necrotic and (B) non-necrotic regions of shRNA-HIF-1 $\alpha$ 4T1 tumors; (C) quantification of HIF-1 $\alpha$ IHC score (addition of intensity score and positive signal area). $n=9$; scale bar: $100 \mu \mathrm{m}$. (D) Reduced relative mRNA expressions of various angiogenesis-associated factors in shRNA-HIF-1 $\alpha$ 4T1 cancer cells cultured in the hypoxic condition $\left(1 \% \mathrm{O}^{2}\right) . n=6$. (E) Immunoblotting for HIF-1 $\alpha$, showing reduced HIF-1 $\alpha$ protein level in Lentivirus-shRNA-HIF-1 $\alpha$ 4T1 cancer cells cultured in hypoxic condition $\left(1 \% \mathrm{O}^{2}\right)$ in vitro. $100 \mu \mathrm{g}$ cellular protein was loaded on each lane. (F) Staining for fitc-conjugated dextran (green, $70 \mathrm{kD}$ ) and CD31 (red) in 4T1 tumors transfected with Lentivirus-shRNA-HIF-1 $\alpha$ or Lentivirus-shRNA-Con. Before extraction, fitc-conjugated dextran was intravenously injected into the tumor-bearing mice for observation of vascular leakage. NR indicates necrotic tumor region. Scale bar: $100 \mu \mathrm{m}$. (G and $\mathbf{H})$ Quantification of $(\mathrm{G})$ detran $^{+}$area leaking outside the tumor lumen and $(\mathrm{H})$ microvessel density (No. of vessels per $\mathrm{mm}^{2}$ ) in 4T1 tumors transfected with either Lentivirus-shRNA-HIF-1 $\alpha$ or Lentivirus-shRNA-Con $(n=10)$. Quantitative data are indicated as mean \pm SEM. ${ }^{*} p<0.05 ;{ }^{* *} p<0.01 ; * * * p<0.001$; ns indicates no significant $(P>0.05)$. 
a mature vasculature is the fundamental basis for efficient blood transportation, this result may give explanation to the elevated blood perfusion. Taken together, our data warrant further studies to elucidate the mechanism underlying the effects of metformin on promoting tumor vascular maturity.

Previous studies were mainly focused on the direct effects of metformin on tumor cells or endothelial cells [23, 31]. A growing number of studies started to confirm that metformin could benefit patients via indirect mechanisms [10, 32, 33]. Our results provide a novel insight into the indirect mechanistic profile leading to inhibited angiogenesis and ameliorated microenvironment by metformin. Additionally, inhibition of AAFs, including VEGF, FGF-2, PDGF-B and PlGF, expands our understanding of angiogenic suppression by metformin, because previous article mainly concentrated in the involvement of the downstream VEGF [10]. These changes of AAFs are closely associated with reduced HIF$1 \alpha$ protein level, which results from ameliorated hypoxia. Moreover, metformin was reported to directly reduce HIF-1 $\alpha$ protein level in normoxia $[10,34]$. This is further supported by the fact that metformin decreased angiogenic ability of vessels even in non-hypoxia region.

Our results indicate that metformin can substantially inhibit tumor angiogenesis by ameliorating tumor hypoxia that has potential therapeutic implications. First, according to the knowledge of tumor response to radiotherapy, metformin-induced amelioration of tumor hypoxia would increase tumor sensitivity to radiotherapy $[13,35]$. Second, the reprogramed hypoxic microenvironment would benefit patients due to reduced therapeutic resistances to chemodrug, immune therapy and anti-angiogenic treatment $[10,32,36,37]$. Third, novel strategies to overcome the barrier of hypoxia in clinical treatment are in dire need, and the anti-diabetic metformin may represent as a workable and safe approach. Fourth, biomarker should be incorporated to predict for patient response to metformin pretreatment. Fifth, angiogenesis-dependent tumors are responsive to metformin, but less has been known about angiogenesis-independent tumors.

In summary, hypoxia-mediated aberrant and excessive angiogenesis was significantly inhibited by metformin administration, which provides a novel rationale for metformin as a new treatment for malignant tumors.

\section{MATERIALS AND METHODS}

\section{Cells}

Mouse breast carcinoma 4T1 cell was obtained from the American Type Culture Collection (ATCC, Manassas, VA) and cultured with Dulbecco's modified Eagle's

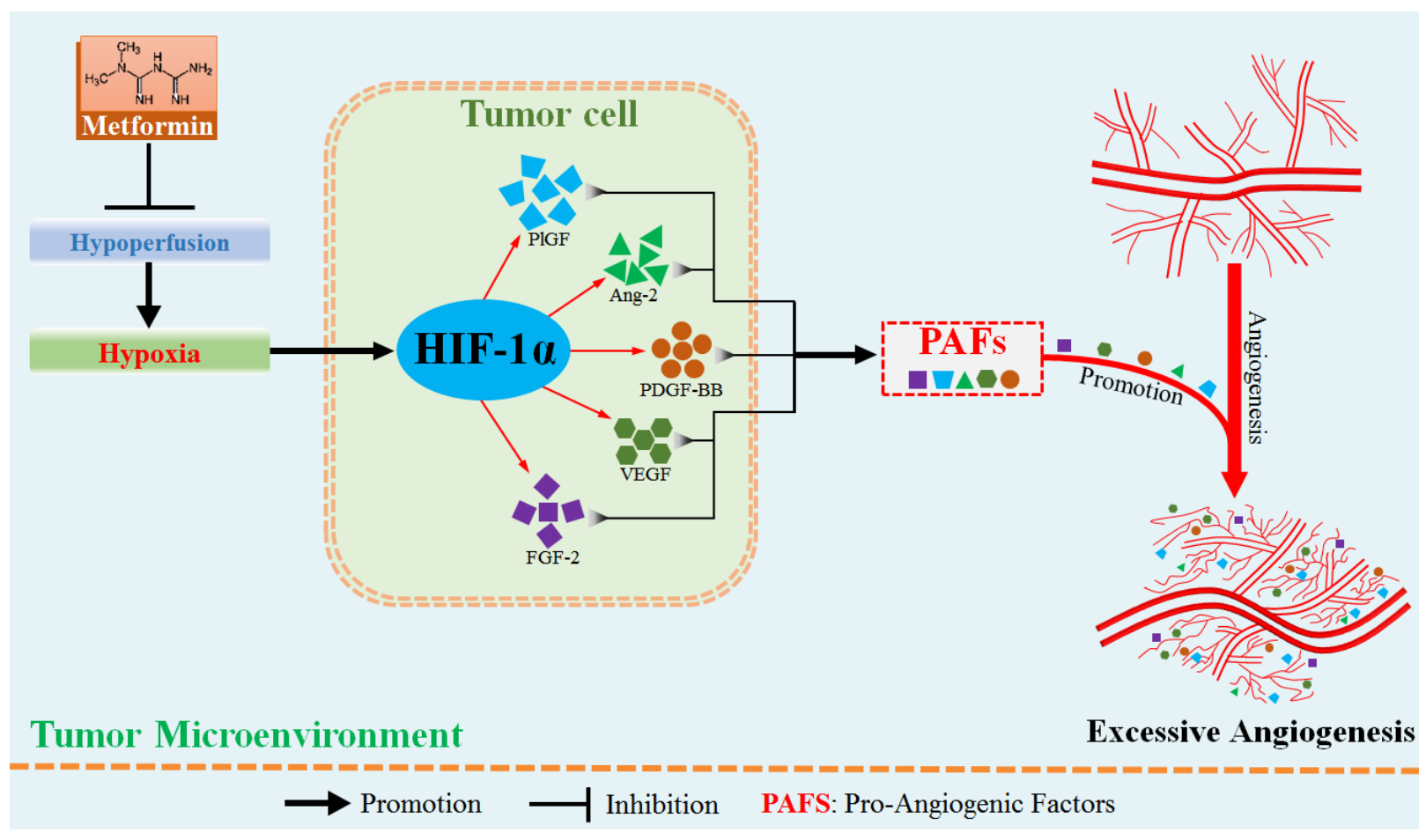

Figure 7: Metformin administration suppressed hypoxia-induced abnormally excessive angiogenesis by ameliorating tumor hypoperfusion. In the hypoxic region adjacent to tumor necrosis, hypoperfusion results in aberrant angiogenesis via hypoxiainduced increase in HIF-1 $\alpha$ protein level. HIF-1 $\alpha$ protein has the potential to up-regulate multiple pro-angiogenic factors, thus greatly enhancing the abilities of sprouting and branching of tumor vessels. Metformin suppresses the abnormally aberrant angiogenesis in tumor by inhibiting HIF-1 $\alpha$-induced expressions of pro-angigenic factors via increase of tumor perfusion. 
medium (DMEM, Invitrogen) supplemented with 10\% fetal bovine serum (FBS, Invitrogen) in an atmosphere of $5 \% \mathrm{CO}^{2}$ and $95 \%$ room air at $37^{\circ} \mathrm{C}$. Hypoxic culture condition was realized by using a hypoxia incubator chamber that could reduce the $\mathrm{O}^{2}$ to $1 \%$.

\section{Generation of HIF-1 $\alpha$ knock-down cells}

Mouse lentiviral shRNA-HIF-1 $\alpha$ and vector shRNA constructs were purchased from SantaCruz (sc35562-V; sc-108080). Vector shRNA lentiviral particle containing scrambled shRNA sequence was used as a negative control. 4T1 cells were transduced with in a mixture of complete medium and Polybrene (Sigma) at a final concentration of $8 \mu \mathrm{g} / \mathrm{ml}$. Transduced cells were finally selected by $2.5 \mu \mathrm{g} / \mathrm{ml}$ puromycin (Cayman). Serial dilution and HIF-1 $\alpha$ expression screen by western blot were performed under $250 \mu \mathrm{M} \mathrm{CoCl}_{2}$ (Sigma) treatment.

\section{Animal models}

All animal experimental protocols were approved by the Ethical Committee and the Institutional Animal Care and Use Committee of Xi' an Jiaotong University. Briefly, $1 \times 10^{6}$ untransfected $4 \mathrm{~T} 1$ cells or $4 \mathrm{~T} 1$ cells with either lentiviral shRNA-HIF-1 $\alpha$ or shRNA-Control was injected into the fat pad of 4 th breast of 6-8 weeks old female BALB/C mice for establishment of an orthotopical breast cancer model. For metformin administration, metformin was advancedly added to drinking water [10]. 28 days after inoculation, mice were sacrificed by abdominal injection of $20 \%$ ethylcarbamate and the whole tumor tissue was extracted.

\section{Immunoblotting}

Protein concentration was determined by Protein Assay Kit (Bio-Rad). For detection of HIF-1 $\alpha, 100 \mu \mathrm{g}$ proteins were loaded on each lane and separated by $10 \%$ SDS-PAGE. Antibodies for HIF-1 $\alpha$ (Abcam), VEGF (ProteinTech) and FGF-2 (Sangon Biotech) were used at a dilution of 1:750, 1:2000 and 1: 4000, respectively, and followed by HRP-conjugated secondary antibody (ProteinTech). A chemiluminescence imaging system was used for quantification of protein expression (Bio-Rad).

\section{Real-time PCR}

To detect mRNA levels of AAFs, a standardized real-time PCR was performed by using SYBR green Master Mix (Takara) according the manufacturer's instructions. All PCR primers used in the present study were listed in Supplementary Table 1.

\section{Vascular leakage, perfusion and tumor hypoxia}

For observation of vascular leakage, $100 \mu \mathrm{l}$ fitcconjugated dextran (100 mg/ml, $70 \mathrm{kDa}$; Sigma) was injected into the tail vein of $\mathrm{BALB} / \mathrm{c}$ mice $30 \mathrm{~min}$ before sacrifice. Tumors were fixed in $4 \%$ paraformaldehyde (PFA) for $8 \mathrm{~h}$, immersed in 30\% sucrose, embedded in OCT and finally cut into $6 \mu \mathrm{m}$ sections. The green fitc signal outside the vascular wall (stained with PEconjugated CD31 antibody, Biolegend) was considered as the leaked dextran. For determination of vascular perfusion and tumor hypoxia in the same area, pimonidazole (PIMO, Cayman) and TRITC-conjugated lectin (Vector Lab) were intravenously injected into the same mouse $2 \mathrm{~h}$ and $15 \mathrm{~min}$ before extraction, respectively. The advancedly prepared tumor sections were then incubated with the mixture of APC-conjugated anti-PIMO antibody (Hypoxyprobe), fitcconjugated anti-CD31 antibody and 5\% BSA overnight.

\section{Immunofluorescence and immunohistochemistry}

For immunohistochemistry, tumor tissues were fixed in $10 \%$ formaldehyde solution, embedded with paraffin and cut into 4-5 $\mu \mathrm{m}$ thick sections. A standard immunohistochemistry staining method was applied for observation of the change of expressions of angiogenesisassociated factors in peri-necrotic region [15]. All antibody applied could be found in Supplementary Table 1 . The result of whole section was finally recorded by using a slide-scanner (Leica). Tumor regions were divided to NR, PNR, TR and NNR according to the histological appearance. NR is the avascular tumor region, where tissue cells are engaged in nuclear condensation and fragmentation. PNR is the excessively vascularized region adjacent to $\mathrm{NR}$, where the average vascular branches per vessels is always higher than 5, whilst that of NNR is less than 3. TR locates between PNR and NNR.

To observe vascular pericyte coverage, 4\% PFAfixed tumor tissue was cut into $40 \mu \mathrm{m}$ sections. Sections were incubated with the mixture of anti-CD31 antibody (Abcam) and anti- $\alpha$-SMA antibody (Boster) for $24 \mathrm{~h}$ at $4^{\circ} \mathrm{C}$, followed by staining with appropriate fluorescently conjugated secondary antibodies (Invitrogen). Nuclei were counterstained by DAPI $(5 \mu \mathrm{g} / \mathrm{mL})$ for 15 min at room temperature before imaging. Images of multi-layers (4-5 $\mu \mathrm{m}$ per layer) were recorded by using a confocal laser scanning microscopy (Leica). 3D-reconstrution of CD31/ $\alpha$-SMA fluorescent signaling was performed by using the software of LAS AF Lite (Leica).

\section{Statistical analysis}

Quantitative data were represented as mean \pm SEM. Two-way ANOVA, one-way ANOVA and Student's $t$ test were used to perform statistical test. Spearman analysis was performed for determination of the linear correlation between MVD of different tumor regions or MVD and necrosis. $* P<0.05, * * P<0.01, * * * P<0.001$; ns indicates no significance. 


\section{Abbreviations}

-SMA: alpha smooth muscle actin; AAFs: angiogenesis-associated factors; Ang-1: angiopoietin-1; Ang-2: angiopoietin-2; ATCC: American Type Culture Collection; DMEM: dulbecco modified eagle medium; FBS: fetal bovine serum; FGF-2: fibroblast growth factor-2; HER2: human epidermal receptor 2; HIF-1 $\alpha$ : hypoxia inducible factor 1 alpha; HRP: horseradish peroxidase; MVD: microvessel density; NNR: non-necrotic region; PDGF-B: platelet-derived growth factor-B; PIMO: pimonidazole; PlGF: placental growth factor; PNR: perinecrotic region; SDS-PAGE: sodium dodecyl sulfate polyacrylamide gel electrophoresis; shRNA: small hairpin RNA; TR: transitional region; VSMCs: vascular smooth muscle cells; TRITC: tetramethyl rhodamin isothiocyanate; VEGF: vascular endothelial growth factor.

\section{Author contributions}

Conception and design: P. J. Liu, S.Y. Lu, J.C. Wang, G.Y. Li; Analysis and interpretation: P. J. Liu, S.Y. Lu, J. C. Wang, G. Y. Li, P. P. Li, X. Sun, W. M. Li, Y. Li; Data collection: S.Y. Lu, J. C. Wang, G. Y. Li; Writing the Article: P. J. Liu, S.Y. Lu, J. C. Wang; Critical revision of the article: J. C. Wang, Y. Li, G. Y. Li, W. M. Li; Final approval of the article: P. J. Liu, S.Y. Lu, J. C. Wang, G. Y. Li; Statistical analysis: P. P. Li, Y. Li, G.Y. Li, X. Sun; Obtained funding: P. J. Liu, S.Y. Lu, J.C. Wang, G.Y. Li; Overall responsibility: P. J. Liu.

\section{ACKNOWLEDGMENTS}

We appreciate the assistance provided by Pan $\mathrm{Li}$ and Mei Xue in sample preparation and immunofluorescence.

\section{CONFLICTS OF INTEREST} interest.

The authors declare that they have no competing

\section{FUNDING}

This work was financially supported by Grants from the National Natural Science Foundation of China (No.81272342, No.81472747, No.81602638 and No.81602502), Science and Technology Program of Shaanxi Province (No.2016SF196), Nature Science Foundation of Shaanxi Province (No.2016JM8125), and College Scientific Research Foundation of Xi'an Jiaotong University (No. xjj2016104).

\section{REFERENCES}

1. Carmeliet P. Angiogenesis in health and disease. Nat Med. 2003; 9:653-60.
2. Shojaei F. Anti-angiogenesis therapy in cancer: current challenges and future perspectives. Cancer Lett. 2012; 320:130-37.

3. Höckel M, Schlenger K, Aral B, Mitze M, Schäffer U, Vaupel P. Association between tumor hypoxia and malignant progression in advanced cancer of the uterine cervix. Cancer Res. 1996; 56:4509-15.

4. Shojaei F, Zhong C, Wu X, Yu L, Ferrara N. Role of myeloid cells in tumor angiogenesis and growth. Trends Cell Biol. 2008; 18:372-78.

5. Pàez-Ribes M, Allen E, Hudock J, Takeda T, Okuyama H, Viñals F, Inoue M, Bergers G, Hanahan D, Casanovas O. Antiangiogenic therapy elicits malignant progression of tumors to increased local invasion and distant metastasis. Cancer Cell. 2009; 15:220-31.

6. Ben Sahra I, Le Marchand-Brustel Y, Tanti JF, Bost F. Metformin in cancer therapy: a new perspective for an old antidiabetic drug? Mol Cancer Ther. 2010; 9:1092-99.

7. Martin MJ, Hayward R, Viros A, Marais R. Metformin accelerates the growth of BRAF V600E-driven melanoma by upregulating VEGF-A. Cancer Discov. 2012; 2:344-55.

8. Pollak M. Metformin and other biguanides in oncology: advancing the research agenda. Cancer Prev Res (Phila). $2010 ; 3: 1060-65$.

9. Pollak MN. Investigating metformin for cancer prevention and treatment: the end of the beginning. Cancer Discov. 2012; 2:778-90.

10. Wang J, Li G, Wang Y, Tang S, Sun X, Feng X, Li Y, Bao G, Li P, Mao X. Suppression of tumor angiogenesis by metformin treatment via a mechanism linked to targeting of HER2/HIF-1 $\alpha$ /VEGF secretion axis. Oncotarget. 2015; 6:44579-44592. https://doi.org/10.18632/oncotarget.6373.

11. Tan BK, Adya R, Chen J, Farhatullah S, Heutling D, Mitchell D, Lehnert H, Randeva HS. Metformin decreases angiogenesis via NF-kappaB and Erk1/2/Erk5 pathways by increasing the antiangiogenic thrombospondin- 1 . Cardiovasc Res. 2009; 83:566-74.

12. Arany Z, Foo SY, Ma Y, Ruas JL, Bommi-Reddy A, Girnun G, Cooper M, Laznik D, Chinsomboon J, Rangwala SM, Baek KH, Rosenzweig A, Spiegelman BM. HIFindependent regulation of VEGF and angiogenesis by the transcriptional coactivator PGC-1alpha. Nature. 2008; 451:1008-12. agr.

13. Zannella VE, Dal Pra A, Muaddi H, McKee TD, Stapleton S, Sykes J, Glicksman R, Chaib S, Zamiara P, Milosevic M, Wouters BG, Bristow RG, Koritzinsky M. Reprogramming metabolism with metformin improves tumor oxygenation and radiotherapy response. Clin Cancer Res. 2013; 19:6741-50.

14. Tao K, Fang M, Alroy J, Sahagian GG. Imagable 4T1 model for the study of late stage breast cancer. BMC Cancer. 2008; $8: 228$.

15. Krohn A, Song YH, Muehlberg F, Droll L, Beckmann C, Alt E. CXCR4 receptor positive spheroid forming cells are 
responsible for tumor invasion in vitro. Cancer Lett. 2009; 280:65-71.

16. Shimizu S, Eguchi Y, Kamiike W, Itoh Y, Hasegawa J, Yamabe K, Otsuki Y, Matsuda H, Tsujimoto Y. Induction of apoptosis as well as necrosis by hypoxia and predominant prevention of apoptosis by Bcl-2 and Bcl-XL. Cancer Res. 1996; 56:2161-66.

17. Raza A, Franklin MJ, Dudek AZ. Pericytes and vessel maturation during tumor angiogenesis and metastasis. Am J Hematol. 2010; 85:593-98.

18. Hellström M, Kalén M, Lindahl P, Abramsson A, Betsholtz C. Role of PDGF-B and PDGFR-beta in recruitment of vascular smooth muscle cells and pericytes during embryonic blood vessel formation in the mouse. Development. 1999; 126:3047-55.

19. Jain RK, Booth MF. What brings pericytes to tumor vessels? J Clin Invest. 2003; 112:1134-36.

20. Kyzas PA, Stefanou D, Batistatou A, Agnantis NJ. Hypoxiainduced tumor angiogenic pathway in head and neck cancer: an in vivo study. Cancer Lett. 2005; 225:297-304.

21. Hiraga T, Kizaka-Kondoh S, Hirota K, Hiraoka M, Yoneda T. Hypoxia and hypoxia-inducible factor-1 expression enhance osteolytic bone metastases of breast cancer. Cancer Res. 2007; 67:4157-63.

22. Dallaglio K, Bruno A, Cantelmo AR, Esposito AI, Ruggiero L. Recchioni S, Calleri A, Bertolini F, Pfeffer U, Noonan DM. Paradoxic effects of metformin on endothelial cells and angiogenesis. Carcinogenesis. 2014.

23. Fukumura D, Jain RK. Tumor microenvironment abnormalities: causes, consequences, and strategies to normalize. J Cell Biochem. 2007; 101:937-49.

24. Jain RK. Normalizing tumor microenvironment to treat cancer: bench to bedside to biomarkers. J Clin Oncol. 2013; 31:2205-18.

25. Karar J, Maity A. Modulating the tumor microenvironment to increase radiation responsiveness. Cancer Biol Ther. 2009; 8:1994-2001.

26. Juan TY, Roffler SR, Hou HS, Huang SM, Chen KC, Leu YL, Prijovich ZM, Yu CP, Wu CC, Sun GH, Cha TL. Antiangiogenesis targeting tumor microenvironment synergizes glucuronide prodrug antitumor activity. Clin Cancer Res. 2009; 15:4600-11.

27. Rini BI, Michaelson MD, Rosenberg JE, Bukowski RM, Sosman JA, Stadler WM, Hutson TE, Margolin K, Harmon CS, DePrimo SE, Kim ST, Chen I, George DJ. Antitumor activity and biomarker analysis of sunitinib in patients with bevacizumab-refractory metastatic renal cell carcinoma. J Clin Oncol. 2008; 26:3743-48.
28. Bridges E, Harris AL. Vascular-promoting therapy reduced tumor growth and progression by improving chemotherapy efficacy. Cancer Cell. 2015; 27:7-9.

29. Wong PP, Demircioglu F, Ghazaly E, Alrawashdeh W, Stratford MR, Scudamore CL, Cereser B, CrnogoracJurcevic T, McDonald S, Elia G, Hagemann T, Kocher HM, Hodivala-Dilke KM. Dual-action combination therapy enhances angiogenesis while reducing tumor growth and spread. Cancer Cell. 2015; 27:123-37.

30. Esfahanian N, Shakiba Y, Nikbin B, Soraya H, MalekiDizaji N, Ghazi-Khansari M, Garjani A. Effect of metformin on the proliferation, migration, and MMP-2 and -9 expression of human umbilical vein endothelial cells. Mol Med Rep. 2012; 5:1068-74.

31. Ding L, Liang G, Yao Z, Zhang J, Liu R, Chen H, Zhou Y, Wu H, Yang B, He Q. Metformin prevents cancer metastasis by inhibiting M2-like polarization of tumor associated macrophages. Oncotarget. 2015; 6:36441-36455. https:// doi.org/10.18632/oncotarget.5541.

32. Isoda K, Young JL, Zirlik A, MacFarlane LA, Tsuboi N, Gerdes N, Schönbeck U, Libby P. Metformin inhibits proinflammatory responses and nuclear factor-kappaB in human vascular wall cells. Arterioscler Thromb Vasc Biol. 2006; 26:611-17.

33. Tadakawa M, Takeda T, Li B, Tsuiji K, Yaegashi N. The antidiabetic drug metformin inhibits vascular endothelial growth factor expression via the mammalian target of rapamycin complex 1/hypoxia-inducible factor-1alpha signaling pathway in ELT-3 cells. Mol Cell Endocrinol. 2014.

34. Choi YW, Lim IK. Sensitization of metformin-cytotoxicity by dichloroacetate via reprogramming glucose metabolism in cancer cells. Cancer Lett. 2014; 346:300-08.

35. Eikawa S, Nishida M, Mizukami S, Yamazaki C, Nakayama E, Udono H. Immune-mediated antitumor effect by type 2 diabetes drug, metformin. Proc Natl Acad Sci USA. 2015; 112:1809-14.

36. Dilman VM, Anisimov VN. Potentiation of antitumor effect of cyclophosphamide and hydrazine sulfate by treatment with the antidiabetic agent, 1-phenylethylbiguanide (phenformin). Cancer Lett. 1979; 7:357-61.

37. Fischer C, Jonckx B, Mazzone M, Zacchigna S, Loges S, Pattarini L, Chorianopoulos E, Liesenborghs L, Koch M, De Mol M, Autiero M, Wyns S, Plaisance S, et al. AntiPIGF inhibits growth of VEGF(R)-inhibitor-resistant tumors without affecting healthy vessels. Cell. 2007; 131:463-75. 